\title{
Machine-Washable Conductive Silk Yarns with a Composite Coating of Ag Nanowires and PEDOT:PSS
}

\author{
Byungil Hwang, *, Anja Lund, ${ }^{\ddagger}$ Yuan Tian, Sozan Darabi, and Christian Müller*
}

Cite This: ACS Appl. Mater. Interfaces 2020, 12, 27537-27544

Read Online

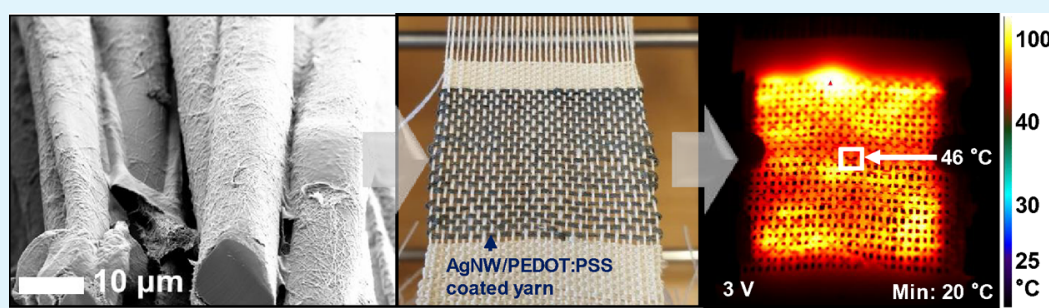

ABSTRACT: Electrically conducting fibers and yarns are critical components of future wearable electronic textile (e-textile) devices such as sensors, antennae, information processors, and energy harvesters. To achieve reliable wearable devices, the development of robust yarns with a high conductivity and excellent washability is urgently needed. In the present study, highly conductive and machine-washable silk yarns were developed utilizing a Ag nanowire and PEDOT:PSS composite coating. Ag nanowires were coated on the silk yarn via a dip-coating process followed by coating with the conjugated polymer:polyelectrolyte complex PEDOT:PSS. The PEDOT:PSS covered the Ag nanowire layers while electrostatically binding to the silk, which significantly improved the robustness of the yarn as compared with the Ag nanowire-coated reference yarns. The fabricated conductive silk yarns had an excellent bulk conductivity of up to $\sim 320 \mathrm{~S} / \mathrm{cm}$, which is largely retained even after several cycles of machine washing. To demonstrate that these yarns can be incorporated into e-textiles, the conductive yarns were used to construct an all-textile out-ofplane thermoelectric device and a Joule heating element in a woven heating fabric.

KEYWORDS: washing machine proof, conductive silk yarn, Ag nanowire, PEDOT:PSS, nanocomposite

\section{INTRODUCTION}

Current developments toward ubiquitous wearable electronic devices, for example, for health monitoring, communication, and entertainment ${ }^{1-4}$ have resulted in a growing interest in combining such devices with textile materials that are worn every day. ${ }^{5,6}$ Electronic textiles (e-textiles) have already been shown to function as sensors, actuators, memory devices, energy harvesters, and energy storage devices. ${ }^{7-11}$ Electronic functionality can be imparted to textiles by several means, for example, printing or coating allow for versatile patterning of the material. ${ }^{12-15}$ However, these methods tend to result in a material, which is sensitive to wear and abrasion. To enable the practical use of e-textiles in everyday life, the material needs to be robust and able to withstand wash and wear. Electrically conducting yarns enable the manufacture of e-textiles without the loss of the intrinsic advantages of textile materials, including softness, flexibility, stretchability, and breathability. Access to conducting yarns as an e-textile component also allows for the design of intricate patterns in textiles via embroidery, jacquard weaving, or knitting, thereby providing a high degree of freedom in the design of wearable electronics. ${ }^{7,16}$

Several types of conductive fibers and yarns have been developed at this point, including solid metal filaments, ${ }^{17,18}$ fibers covered with metal-based materials such as metal nanoparticles or nanofibers, ${ }^{19-24}$ or deposited metal or metal oxide films, ${ }^{25-27}$ as well as fibers where conductivity is imparted by conjugated polymers ${ }^{28-30}$ or carbon-based materials, e.g., carbon nanotubes (CNTs) or graphene. ${ }^{31,32}$ We observe that there is a trade-off between conductivity and wearer comfort, where the coating imparts a high conductivity but also makes a textile less comfortable to wear. For example, copper filaments have an excellent conductivity of $6 \times 10^{5} \mathrm{~S} /$ $\mathrm{cm}$, but they are unsuitable for wearable applications because of their limited stretchability. ${ }^{17}$ Although fibers dyed with conducting polymers can be integrated into wearable devices, the conductivity of those fibers is limited to $\sim 70 \mathrm{~S} / \mathrm{cm}$, which must be improved further. ${ }^{16}$ Fibers covered by metal ${ }^{28-30}$ or carbon-based materials ${ }^{31,32}$ showed higher conductivities than those fabricated with conducting polymers but such fibers tend to lose their conductivity during machine washing.

Received: March 6, 2020

Accepted: May 22, 2020

Published: May 22, 2020 


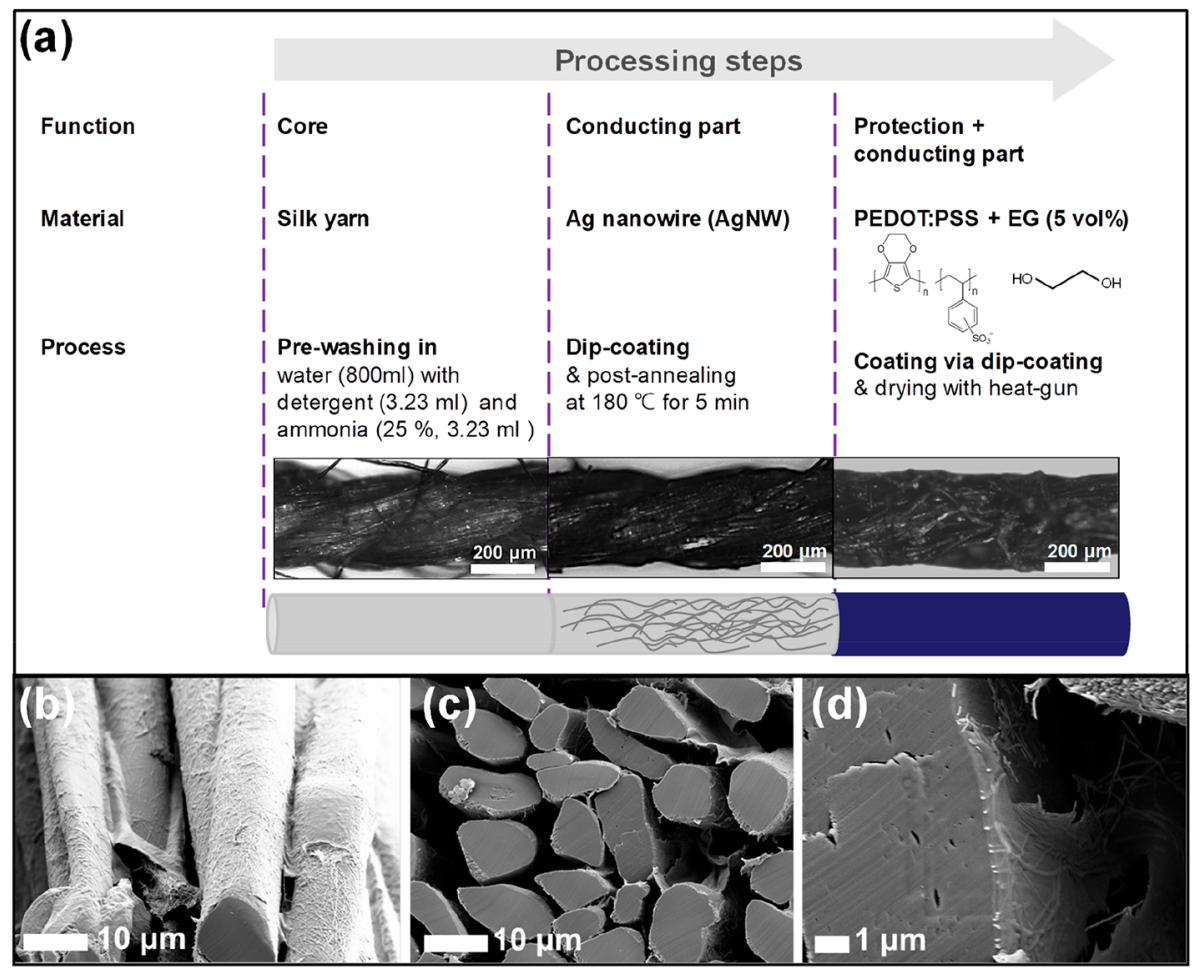

Figure 1. (a) Schematic and description of the preparation of conductive silk yarns with an Ag nanowire/PEDOT:PSS composite coating. (b) Top view and (c, d) cross-sectional SEM images of individual filaments that make up the conductive silk yarns.

Here, we propose that a way forward is to combine $\mathrm{Ag}$ nanowires with a conducting polymer. Ag nanowires can be synthesized in large volumes through a polyol reduction solution process and can impart conductivity to textile fibers and yarns via a simple dip-coating procedure. ${ }^{33-36}$ In addition, a coating of Ag nanowires is composed of a network structure that can accommodate deformation, thereby resulting in excellent mechanical robustness. ${ }^{37-40}$ We find that a layer of Ag nanowires on silk fibers forms a highly conductive network. After the addition of the conducting polymer:polyelectrolyte complex poly(3,4-ethylene dioxythiophene):poly(styrene sulfonate) (PEDOT:PSS), ${ }^{7,16,41,42}$ the yarns display both excellent mechanical robustness under deformation, and can be machine-washed without loss of conductivity. Since $\mathrm{Ag}$ nanowires are required to cover only the surface of the yarns, and a coverage of $\sim 74 \%$ is sufficient to impart conductivity, the material consumption is much lower than that of their metal particle or nanowire-embedded polymer fiber competitors, where more than $\sim 30$ vol $\%$ of metal particles or nanowires are needed to form conducting paths through the entire volume of fibers. $^{43-47}$

We now demonstrate a machine-washable Ag nanowire/ PEDOT:PSS-based conductive silk yarn with an outstanding conductivity of up to $\sim 320 \mathrm{~S} / \mathrm{cm}$ and excellent washability compared to that of $\mathrm{Ag}$ nanowire-only coated yarns. To prepare these yarns we developed a sequential batch coating process that involves repeated coating with Ag nanowires followed by coating with PEDOT:PSS, which ingresses into individual silk filaments and strongly binds through electrostatic interactions. ${ }^{7,16}$ To demonstrate that our yarns can be readily used to construct wearable electronics, we fabricated an all-textile out-of-plane thermoelectric device that featured a ptype leg composed of the PEDOT:PSS-coated yarn and interconnects composed of the Ag nanowires/PEDOT:PSS- coated yarn. Furthermore, we designed a woven heating fabric using our conductive yarn as the Joule heating element.

\section{EXPERIMENTAL SECTION}

2.1. Materials. Ag nanowire solution ( $0.5 \mathrm{wt} \%$ in isopropyl alcohol, Merck), degummed (i.e., the natural sericin has been removed) silk yarn (Aurora Silk, diameter: $\sim 236 \mu \mathrm{m}$ ), PEDOT:PSS dispersion (PH1000, Heraeus, $850 \mathrm{~S} / \mathrm{cm}, \sim 1.3 \mathrm{wt} \%$ of solid content), ethylene glycol (EG) (Sigma-Aldrich), silk detergent (Zenit), and 4 $\mathrm{g} / \mathrm{dm}^{3}$ ammonia (25\%, Merck Millipore) were all used as received. According to the certification of analysis from Merck, the $\mathrm{Ag}$ nanowires have an average diameter and length of $40 \pm 5 \mathrm{~nm}$ and 35 $\pm 5 \mu \mathrm{m}$ (Figure S6), respectively. The PEDOT:PSS dispersion was prepared as a mixture of PEDOT:PSS and 5 vol \% EG.

2.2. Fiber and Textile Preparation. Prewashing of the silk was conducted to remove any contamination on the surface by stirring at $200 \mathrm{rpm}$ in a mixed solution of $800 \mathrm{~mL}$ deionized water, $3.23 \mathrm{~mL}$ silk detergent, and $3.23 \mathrm{~mL}$ ammonia for $30 \mathrm{~min}$ at $40{ }^{\circ} \mathrm{C}$. The silk content in the mixed washing solution was $\sim 0.35 \mathrm{wt} \%$. The washed silk yarn was dried at room temperature for $24 \mathrm{~h}$ before usage. The washed silk yarn was dipped into an $\mathrm{Ag}$ nanowire solution and then baked at $180^{\circ} \mathrm{C}$ for $5 \mathrm{~min}$ in air using a hot plate. The volume ratio of silk in the Ag nanowire solution was $\sim 0.2 \mathrm{vol} \%$, and the wt \% ratio of the silk and the Ag nanowires in the solution was $2.9: 1$. Next, the Ag nanowire-coated silk yarn was coated using the PEDOT:PSS solution by the same dip-coating process as that for the $\mathrm{Ag}$ nanowire coating followed by drying with a heat gun, which heated the air to around $100{ }^{\circ} \mathrm{C}$. The volume ratio of the $\mathrm{Ag}$ nanowire-coated silk in PEDOT:PSS dispersion was $\sim 0.1 \mathrm{vol} \%$. The drying continued until the PEDOT:PSS was resistant to rubbing. The PEDOT:PSS-only coated yarns were fabricated, as described above, leaving out the Ag nanowire coating process. Silk yarns were dipped into the PEDOT:PSS dispersion by the dip-coating process and dried with a heat gun.

A thermoelectric textile was manufactured by hand embroidering through eight layers of felted wool fabric (Wadmal, $\sim 1 \mathrm{~mm}$ thick, 3.2 $\left.\mathrm{g} / \mathrm{dm}^{2}\right)$, which served as a thermal insulator. The thermoelectric p- 
(a)

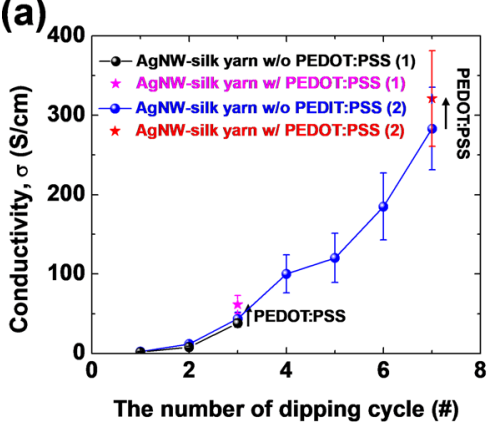

(b)

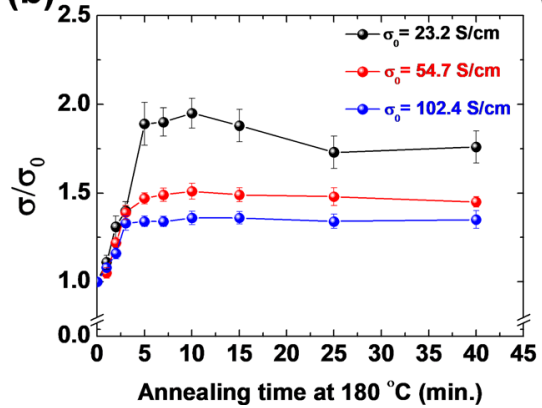

(c)

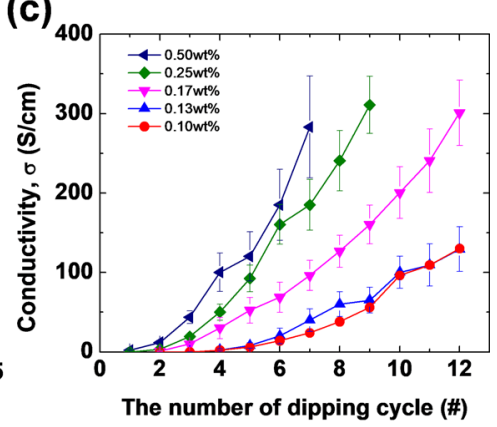

Figure 2. (a) Electrical conductivity of silk yarns as a function of the number of dip-coating cycles in the Ag nanowire solution. (b) Normalized conductivity of the silk yarns after (black) two, (red) three, and (blue) four dipping cycles as a function of the $180{ }^{\circ} \mathrm{C}$ postannealing time. $\sigma_{0}$ indicates the initial conductivity before annealing. (c) Conductivity of the silk yarns as a function of the number of dip-coating cycles in Ag nanowire solutions of different concentrations.

type legs comprised previously reported PEDOT:PSS-coated silk yarns from our group, ${ }^{16}$ and the Ag nanowire/PEDOT:PSS-coated yarns functioned as a connector between the p-type legs. Silver paste (Agar Scientific) was applied to minimize the contact resistance between the thermoelectric leg-pairs.

The Joule heating plain weave fabric was manufactured in a custom-built miniature loom with a flax yarn warp, where the $\mathrm{Ag}$ nanowire/PEDOT:PSS-coated yarns were inserted as the weft.

2.3. Electrical Characterization. The Seebeck coefficient of the yarns was measured at $300 \mathrm{~K}$ by using a digital Seebeck controller (SB1000, MMR Technology) coupled with a temperature controller (K2000, MMR Technology). The length of the yarn used for the Seebeck coefficient measurement was $\sim 5 \mathrm{~mm}$, the thermal load was $1-2 \mathrm{~K}$, and a constantan wire was used as a reference.

To evaluate the thermoelectric effect of our textile device, the textile was placed on a hot plate that was set to different temperatures. A room tempered cooler was placed on top of the textile, and the respective temperatures were measured with two K-type adhesive thermocouples (Omega Engineering). The voltage generated by the module at different temperature gradients was measured using a source meter (Keithley 2400). Machine washing was conducted using a commercial washing machine (Daewoong Morning Calm) with 30 $\mathrm{mL}$ of commercial detergent (Spark, Aekyung Co. Ltd.) in $3 \mathrm{~L}$ of water. The detergent is reported to contain linear alkylbenzenesulfonates, $\alpha$-olefin, zeolite, fatty acid, etc. The three yarns for each condition were put into a mesh laundry bag and then washed in the washing machine with $3 \mathrm{~L}$ of water. Each washing cycle was performed for $20 \mathrm{~min}$ at $30{ }^{\circ} \mathrm{C}$. The mechanical stability was evaluated using a cyclic bending tester (Supporting Information Figure S2). For the tests, yarn samples were attached to a polyimide substrate (125 $\mu \mathrm{m}$ thickness, Dupont) with silver paste, which was then fixed to the bending machine with metal bolts that enabled in situ resistance monitoring. The bending radius was fixed at $1 \mathrm{~mm}$, and each test lasted up to 300,000 cycles with a bending rate of $300 \mathrm{~Hz}$. The resistance was measured in a two-point configuration using a digital multimeter (Agilent 34401A). ${ }^{48-50}$

2.4. Microscopy and Imaging. Field-emission scanning electron microscopy (FESEM, Leo Ultra 55) and optical microscopy (OM, Nikon, Eclipse LV150N) were used to characterize the surface texture of the yarns. To obtain cross-sectional images, the yarns were cooled with liquid nitrogen and then cut with a razor blade. An infrared (IR) camera (FLIR A645sc) was used to take the thermal images.

\section{RESULTS AND DISCUSSION}

Our conducting silk yarns were fabricated using a multistep dip-coating procedure (Figure 1a). The prewashed silk yarn was repeatedly dipped into a 0.5 wt $\% \mathrm{Ag}$ nanowire solution until the desired resistance value was achieved. Next, the $\mathrm{Ag}$ nanowire-coated silk yarn was coated with a PEDOT:PSS/EG water-based dispersion followed by drying with a heat gun. The addition of 5 vol \% EG to the PEDOT:PSS coating formulation has been observed to enhance electrical conductivity and washability. ${ }^{7,16}$ The PEDOT:PSS penetrated both the Ag nanowire layers and the silk yarn, resulting in a yarn with a macroscopically smooth surface (Figure 1a) and a diameter of $\sim 230 \mu \mathrm{m}$. The final yarn diameter was not significantly affected by the number of $\mathrm{Ag}$ nanowire coating cycles but was instead determined by the relatively thick PEDOT:PSS layer. Cross-sectional (Figure $1 \mathrm{~b}-\mathrm{d}$ ) and top view SEM images (Figure S1) reveal that each individual filament in the yarn was covered by a composite coating of $\mathrm{Ag}$ nanowires and a PEDOT:PSS layer.

The final conductivity of the Ag nanowire-based silk yarn can be controlled by the number of dip-coating cycles (Figure $2 \mathrm{a})$. The conductivity was calculated according to $\sigma=L /(R \times$ $A)$, where $L$ is the length of the yarn, $R$ is the resistance in a 2point configuration measured after each dip-coating cycle, and $A$ is the cross-sectional area of the yarn. $L, R$, and $A$ were all measured after each dip-coating cycle. The conductivity of the silk yarns increased almost linearly as the number of $\mathrm{Ag}$ nanowire dip-coating cycles increased, and a conductivity of $\sim 284 \mathrm{~S} / \mathrm{cm}$ was achieved after seven cycles. The addition of PEDOT:PSS further increased the conductivity to $\sim 320 \mathrm{~S} / \mathrm{cm}$ because the conjugated polymer complex increases the amount of conducting material. PEDOT:PSS covers the Ag nanowires and provides additional conducting paths by filling the vacant spaces within the nanowire network. The increase in weight of the silk yarns after seven cycles of Ag nanowire coating and after the final PEDOT:PSS coating was $\sim 0.06$ and $0.19 \mathrm{mg} /$ $\mathrm{cm}$, respectively, which implies that the fraction of PEDOT:PSS $(0.13 \mathrm{mg} / \mathrm{cm})$ in the Ag nanowire/PEDOT:PSS composite coating was larger than that of the Ag nanowires. Overall, the conducting silk yarn was composed of $\sim 0.13 \%$ conducting material by weight. The Seebeck coefficient of the Ag nanowire/PEDOT:PSS-coated silk yarns was $\sim 0.8 \mu \mathrm{V} / \mathrm{K}$, which is similar in magnitude to silver $(-0.2 \mu \mathrm{V} / \mathrm{K}$ as measured on a $300 \mu \mathrm{m}$-thick fine silver wire), albeit of different signs. The PEDOT:PSS-only coated yarn had a Seebeck coefficient of $15.0 \mu \mathrm{V} / \mathrm{K}$. The fact that the Seebeck coefficient of our composite material is close to that of pure silver, i.e., does not abide by the rule of mixtures in composites, further confirms that the majority of current flow occurs through the $\mathrm{Ag}$ nanowire network and not the PEDOT:PSS in the composites.

The electrical conductivity was further enhanced by postannealing at $180{ }^{\circ} \mathrm{C}$ (Figure $2 \mathrm{~b}$ ), a standard procedure 
(a)

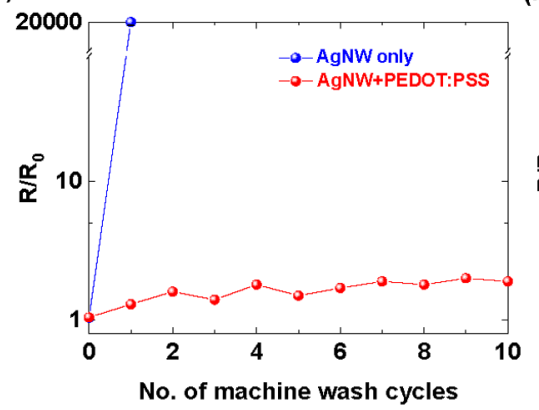

(b)

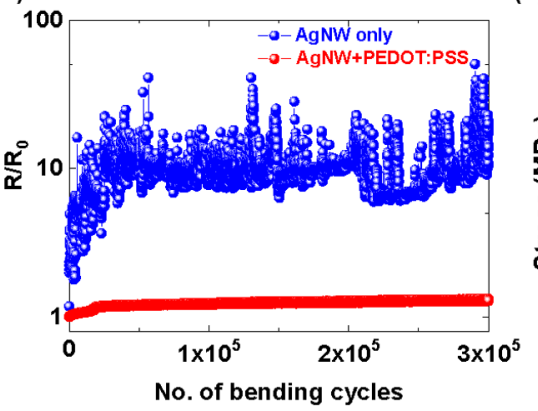

(c)

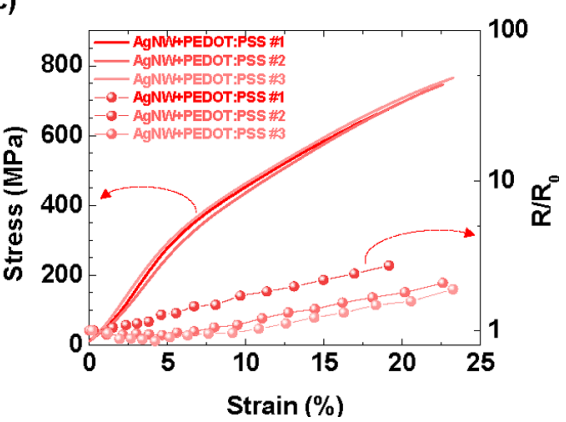

Figure 3. Normalized resistance change of silk yarns with Ag nanowire and Ag nanowire/PEDOT:PSS coatings as a function of (a) number of machine-washing cycles, and (b) number of bending cycles. (c) Stress-strain curves of the silk yarns with the composite coating of Ag nanowire and PEDOT:PSS (solid lines) and the change in normalized resistance measured during tensile tests (symbols). $R_{0}$ is the initial resistance before testing, and $R$ is the resistance measured under washing/bending/tensile tests.
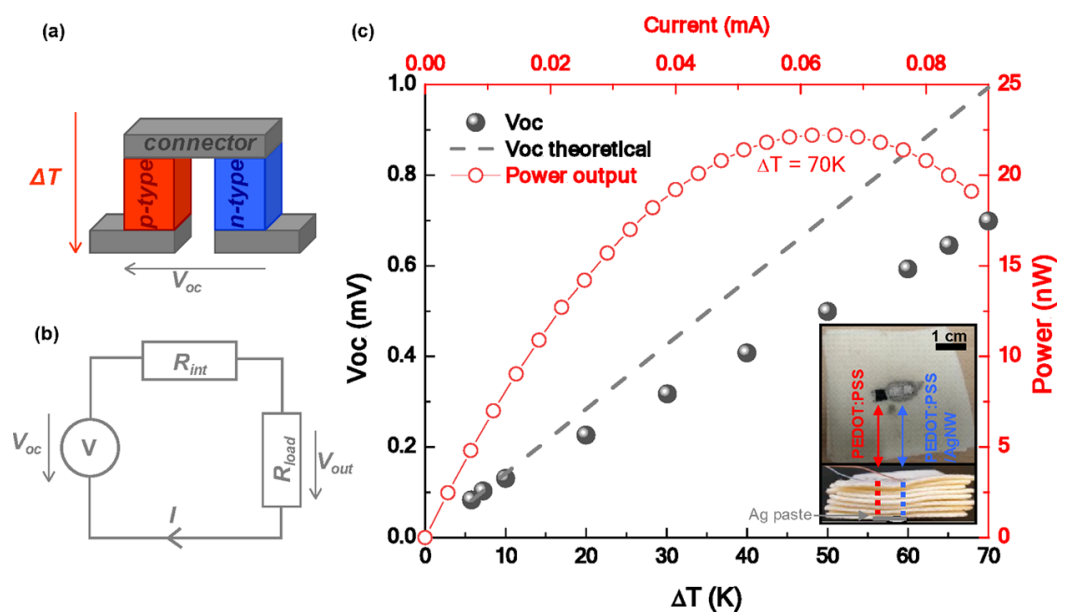

Figure 4. Schematics of (a) a thermocouple and (b) its equivalent electric circuit. (c) Electrical measurement of a thermoelectric textile consisting of a single thermocouple, with the open circuit voltage, $V_{\mathrm{oc}}$ as a function of $\Delta T$, and the power output as a function of the measured current at $\Delta T$ $=70 \mathrm{~K}$. Insets are top and side view photographs of the out-of-plane thermoelectric device with one n-type leg composed of the Ag nanowire/ PEDOT:PSS-coated silk yarn and one p-type leg composed of the PEDOT:PSS-only coated silk yarn.

because the as-synthesized $\mathrm{Ag}$ nanowires are encapsulated by a very thin polyvinylpyrrolidone (PVP) layer that hinders current flow at the junction between the individual nanowires. During synthesis of Ag nanowires, PVP is added to promote anisotropic growth of Ag nanowires along the $<110>$ direction, where the PVP encapsulates the [100] surface of Ag nanowires, allowing silver ions to attach only on the [111] surface. ${ }^{51}$ By annealing at $180{ }^{\circ} \mathrm{C}$, adjacent $\mathrm{Ag}$ nanowires were thermally fused, which reduced the junction resistance and thereby enhanced the conductivity. ${ }^{52}$ The conductivity had saturated after $5 \mathrm{~min}$ of annealing; therefore, we opted to anneal all other samples for 5 min during the fabrication process.

To identify the minimal amount of material that is required to achieve a high electrical conductivity, we explored dipcoating into $\mathrm{Ag}$ nanowire solutions of various concentrations. The use of 0.5 and $0.25 \mathrm{wt} \% \mathrm{Ag}$ nanowire solutions resulted in similar trends with regard to the increase in conductivity (Figure 2c). Only one or two additional coating cycles were required for the yarn dipped in the $0.25 \mathrm{wt} \% \mathrm{Ag}$ nanowire solution to achieve a similar conductivity to that of the yarn repeatedly dipped in the $0.5 \mathrm{wt} \%$ solution. Therefore, for efficient use of material, a 0.25 wt \% Ag nanowire solution could be used instead of a 0.5 wt \% Ag nanowire solution. When the Ag nanowire solution was diluted to a solid content
$<0.25$ wt \%, at least 12 dip-coating cycles were required to achieve a high conductivity; thus, the highly diluted solutions are less efficient.

To examine the degree of machine washability of the $\mathrm{Ag}$ nanowire-based conductive silk yarns, the resistance change of the yarns with the Ag nanowire-only coating and with the optimized Ag nanowire/PEDOT:PSS composite coating was measured after each machine-washing cycle (Figure 3a). The silk yarn with the Ag nanowire-only coating showed a rapid increase in resistance by 4 orders of magnitude after only one washing cycle and became highly resistive after two washing cycles. The Ag nanowire/PEDOT:PSS-coated yarn only doubled its resistance even after ten washing cycles. The washability of the Ag nanowire/PEDOT:PSS-coated yarn was even superior to that of a widely used Ag-plated commercial conductive yarn (Figure S3). The excellent washability of the Ag nanowire/PEDOT:PSS-coated yarn was attributed to a strong electrostatic interaction between the PEDOT:PSS coating and the silk. Conveniently, the low $\mathrm{pH}(\approx 2)$ of the PEDOT:PSS aqueous dispersion makes coating of silk materials relatively straight forward: at this low $\mathrm{pH}$ the silk filament surface will possess a net positive charge (isoelectric point $\mathrm{pH} \approx 4$ ), which provides electrostatic attraction to the negatively charged sulfonate groups of PEDOT:PSS. Moreover the addition of EG to the PEDOT:PSS dispersion results in a 

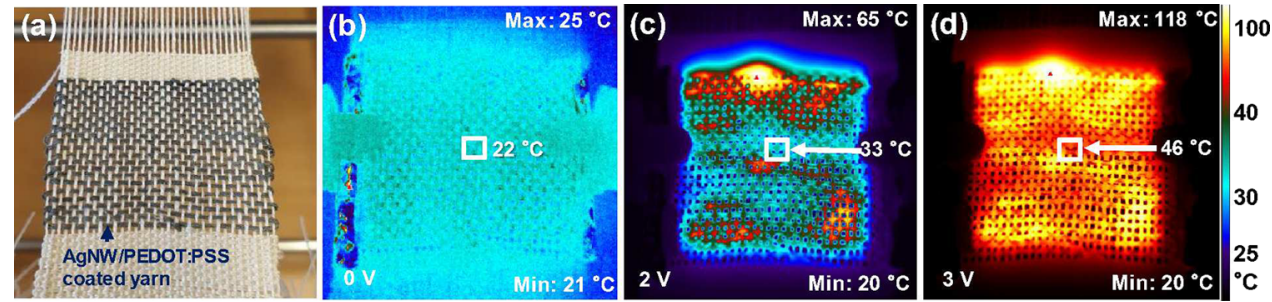

Figure 5. (a) Photograph of woven heating fabric using the Ag nanowire/PEDOT:PSS-coated conductive silk yarn as a Joule heating element, and IR camera images of the heating fabric when applying (b) no voltage, (c) $2 \mathrm{~V}$, and (d) $3 \mathrm{~V}$. The respective IR images were taken no more than $30 \mathrm{~s}$ after the voltage was switched on.

coating that is water-stable after drying, ${ }^{53}$ possibly as a result of hydrogen-bonding between the EG and the PSS components. ${ }^{34}$

The robustness of the Ag nanowire-based conductive silk yarns was further confirmed by cyclic bending fatigue tests, where the samples experienced 300,000 bending cycles, during which we monitored the electrical resistance (Figure $3 \mathrm{~b}$ ). The silk yarn with the Ag nanowire-only coating showed a nearly two orders of magnitude increase in resistance after 300,000 bending cycles, whereas a less than $30 \%$ increase in resistance was observed for the Ag nanowire/PEDOT:PSS-coated conductive yarn. Because the Ag nanowires were only physically adsorbed on the surface of the silk yarn with the Ag nanowire-only coating, they were easily removed by repeated bending or wear. With the addition of the PEDOT:PSS layer, however, the Ag nanowires more tightly adhered to the silk, strongly enhancing the resistance to external mechanical damage. To further understand the electrical/mechanical performance of the conductive silk yarns with the Ag nanowire/PEDOT:PSS composite coating, tensile tests were performed while monitoring the resistance in situ (Figure 3c). The conductive silk yarns display a tensile strength of $\sim 750 \mathrm{MPa}$ with a rupture strain of $\sim 23 \%$ and a linear shape in stress-strain curves. Evidently, the conducting $\mathrm{Ag}$ nanowire network is preserved at least up to the rupture strain with only a twofold increase in resistance at maximum strain.

To demonstrate that our Ag nanowire-based conductive silk yarns can be readily used to construct wearable electronic devices, we fabricated a model textile thermoelectric device for energy harvesting. Owing to the Seebeck effect, a thermoelectric device can generate a voltage when subjected to a temperature gradient. The device base unit is the thermocouple (Figure 4a), which consists of two "legs" composed of one p-type carrier material and one n-type carrier material. The legs are connected electrically in series, typically by the use of a conducting component, e.g., silver paste and thermally in parallel. The open circuit voltage $V_{\text {oc }}$ generated in response to a given temperature gradient $\Delta T$ can be predicted by:

$$
V_{\mathrm{oc}}=\left(\alpha_{\mathrm{p}}-\alpha_{\mathrm{n}}\right) \times \Delta T
$$

where $\alpha_{\mathrm{p}}$ and $\alpha_{\mathrm{n}}$ are the Seebeck coefficients of the $\mathrm{p}$ - and ntype materials, respectively. ${ }^{7}$ The PEDOT:PSS-only coated yarn used in our study has a positive Seebeck coefficient of $15.0 \mu \mathrm{V} / \mathrm{K}$, and the Ag nanowire/PEDOT:PSS-coated yarns display a positive but small Seebeck coefficient of $0.8 \mu \mathrm{V} / \mathrm{K}$. The silver paste, applied laterally on each surface, will experience a very small temperature gradient over its own thickness. The Seebeck coefficient of our used silver paste was measured to be $-0.2 \mu \mathrm{V} / \mathrm{K}$, so we anticipate that it does not influence the voltage produced by our device. We hand embroidered our two yarns through a $\sim 10 \mathrm{~mm}$ thick multilayered wool fabric, to form an out-of-plane thermoelectric device that offers the possibility to be directly incorporated into clothing or upholstery. According to eq 1 , the open circuit voltage $V_{\text {oc }} / \Delta T$ for the one thermocouple in our thermoelectric device was estimated to be $14.2 \mu \mathrm{V} / \mathrm{K}$ (dashed line in Figure 4). The slightly lower generated voltage that we measure is attributed to the presence of thermal contact resistances in our experimental setup, i.e., there is a temperature drop across the interfaces between the textile and the heat source and the cooler, respectively. Therefore, the thermocouple experiences a smaller $\Delta T$ compared to what our external sensors measure.

The thermoelectric device can be represented by an equivalent electric circuit (Figure $4 b$ ) consisting of a voltage supply generating $V_{\mathrm{oc}}$ an internal resistance (in this case constituted by our embroidered textile plus the connecting silver paste) $R_{\text {int }}$ and a load represented by a resistor $R_{\text {load }}$. The power $P_{\text {load }}$ generated can then be calculated as:

$$
P_{\text {load }}=V_{\text {out }} \times I=V_{\text {out }} \times V_{\text {oc }} /\left(R_{\text {load }}+R_{\text {int }}\right)
$$

where $V_{\text {out }}$ is the voltage generated over the load resistance $R_{\text {load }}$, and $I$ is the circuit current. Because the maximum power $P_{\max }$ is found under load matching conditions, i.e., when $R_{\text {load }}=$ $R_{\text {int }}$ and consequently $V_{\text {out }}=V_{\text {oc }} / 2, P_{\max }$ for a certain $\Delta T$ can be predicted by:

$$
P_{\max }=V_{\text {oc }}^{2} /\left(4 R_{\text {int }}\right)
$$

The power output of our device was measured at $\Delta T \approx 70 \mathrm{~K}$ while varying the load currents, and we recorded a maximum power output of $22.3 \mathrm{nW}$. We measured the internal resistance of our thermocouple to be $\sim 10 \mathrm{ohms}$, and so the predicted $P_{\max }=24.7 \mathrm{nW}$ matches the measured generated power. The power generated by our single thermocouple device exceeds that generated by multi-thermocouple devices in several previous reports, ${ }^{55-57}$ which can be largely attributed to the high conductivity of our yarns. The power output decreased as the $\Delta T$ decreased, as shown in Figure $S 8$ in the Supporting Information.

The Ag nanowire/PEDOT:PSS composite-coated yarns were also used to weave a heating fabric to demonstrate the use of our yarns as a Joule heating element. The coated yarns were used as the weft in a plain weave textile (Figure 5a, Figure S7). As this fabric was connected, via crocodile clamps (Figure S7), to a power supply and powered with $2-3 \mathrm{~V}$, its temperature quickly increased (Figure $5 b-d$ ). Without the applied voltage, we measured a maximum temperature of 25 ${ }^{\circ} \mathrm{C}$ on the fabric (center: $22{ }^{\circ} \mathrm{C}$ ) (Figure $5 \mathrm{~b}$ ). Upon the application of $2 \mathrm{~V}$, the temperature rapidly increased to a 
maximum temperature of $64{ }^{\circ} \mathrm{C}$ (center: $33^{\circ} \mathrm{C}$ ) as shown in Figure $5 c$, and an even higher temperature was achieved under the application of $3 \mathrm{~V}$, as shown in Figure $5 \mathrm{~d}$. The respective IR images were taken no more than $30 \mathrm{~s}$ after the voltage was switched on. The natural texture of woven fabrics will influence their electrical properties due to the varying contact resistances between individual yarns in the weave, resulting in "hot spots" and some inhomogeneity in the temperature distribution. The achieved high temperature of the heating fabric upon application of a small voltage clearly shows the potential of our conductive yarns for use as the Joule heating element of a wearable heater.

\section{CONCLUSIONS}

In this study, we developed a process to apply a $\mathrm{Ag}$ nanowire and PEDOT:PSS composite coating to form highly robust conductive silk yarns. The Ag nanowires were coated on silk via a simple dip-coating process and then a PEDOT:PSS dispersion containing 5 vol \% of EG was used to coat the yarn surface. This process was able to utilize the benefits of both the $\mathrm{Ag}$ nanowires and PEDOT:PSS, where the Ag nanowires provided high conductivity, and PEDOT:PSS with EG provided excellent wash and wear resistance. The Ag nanowire/PEDOT:PSS-coated conductive silk yarns showed a high conductivity of up to $\sim 320 \mathrm{~S} / \mathrm{cm}$ and excellent robustness after washing, showing no significant increase in resistance even after machine washing ten times with detergent. To demonstrate the utility of these yarns, a textile-based out-of-plane thermoelectric device was fabricated that incorporated the conductive silk yarn. In addition, a woven heating fabric using the conductive silk yarn as the Joule heating element was successfully fabricated, producing enough heat at an applied voltage of $2-3 \mathrm{~V}$ for wearable heating devices. It can be anticipated that the developed conductive silk yarns with high conductivity and excellent washability will help to accelerate the commercialization of robust wearable electronic devices.

\section{ASSOCIATED CONTENT}

\section{s) Supporting Information}

The Supporting Information is available free of charge at https://pubs.acs.org/doi/10.1021/acsami.0c04316.

SEM images of silk, Ag nanowire-coated silk, Ag nanowire/PEDOT:PSS-coated silk yarn, and PEDOT:PSS-coated silk yarn; schematic and photo of bending tester and samples; comparison of machine washability with a commercial yarn; cross-sectional SEM image of Ag nanowire/PEDOT:PSS-coated yarns; EDX mapping image on the surface of $\mathrm{Ag}$ nanowire/ PEDOT:PSS-coated yarns; SEM image of Ag nanowires; photograph of the miniature loom used to weave the Joule heating fabric, close-up photograph of the Joule heating fabric and a schematic representation of the plain weave fabric (PDF)

\section{AUTHOR INFORMATION}

\section{Corresponding Authors}

Byungil Hwang - School of Integrative Engineering, Chung-Ang University, Seoul 06974, Republic of Korea; (1) orcid.org/ 0000-0001-9270-9014; Email: bihwang@cau.ac.kr

Christian Müller - Department of Chemistry and Chemical Engineering and Wallenberg Wood Science Center, Chalmers
University of Technology, 41296 Göteborg, Sweden; (1) orcid.org/0000-0001-7859-7909; Email: christian.muller@chalmers.se

Authors

Anja Lund - Department of Chemistry and Chemical Engineering, Chalmers University of Technology, 41296 Göteborg, Sweden

Yuan Tian - Department of Chemistry and Chemical Engineering, Chalmers University of Technology, 41296 Göteborg, Sweden

Sozan Darabi - Department of Chemistry and Chemical Engineering and Wallenberg Wood Science Center, Chalmers University of Technology, 41296 Göteborg, Sweden

Complete contact information is available at: https://pubs.acs.org/10.1021/acsami.0c04316

\section{Author Contributions}

${ }^{\ddagger}$ B.H. and A.L. contributed equally to this work.

\section{Notes}

The authors declare no competing financial interest.

\section{ACKNOWLEDGMENTS}

We gratefully acknowledge financial support from the Swedish Research Council through grant no. 2016-06146, the Knut and Alice Wallenberg Foundation through a Wallenberg Academy Fellowship, the European Research Council (ERC) under grant agreement no. 637624, and the Wallenberg Wood Science Center. We also gratefully acknowledge financial support from the National Research Foundation (NRF) of Korea, which is funded by the Ministry of Science, Information and Communications Technology (Grant Nos. NRF2018R1C1B5043900 and NRF-2019K1A3A1A47000624). We thank Alexander Dmitriev (University of Gothenburg) for lending his IR camera. This work was performed in part at the Chalmers Material Analysis Laboratory, CMAL.

\section{REFERENCES}

(1) Shi, J.; Liu, S.; Zhang, L.; Yang, B.; Shu, L.; Yang, Y.; Ren, M.; Wang, Y.; Chen, J.; Chen, W.; Chai, Y.; Tao, X. Smart TextileIntegrated Microelectronic Systems for Wearable Applications. Adv. Mater. 2019, 32, 1901958.

(2) Zhao, S.; Li, J.; Cao, D.; Zhang, G.; Li, J.; Li, K.; Yang, Y.; Wang, W.; Jin, Y.; Sun, R.; Wong, C.-P. Recent Advancements in Flexible and Stretchable Electrodes for Electromechanical Sensors: Strategies, Materials, and Features. ACS Appl. Mater. Interfaces 2017, 9, 1214712164.

(3) Bauer, S.; Bauer-Gogonea, S.; Graz, I.; Kaltenbrunner, M.; Keplinger, C.; Schwödiauer, R. 25th Anniversary Article: A Soft Future: From Robots and Sensor Skin to Energy Harvesters. Adv. Mater. 2014, 26, 149-162.

(4) Someya, T.; Bao, Z.; Malliaras, G. G. The Rise of Plastic Bioelectronics. Nature 2016, 540, 379-385.

(5) Wang, B.; Facchetti, A. Mechanically Flexible Conductors for Stretchable and Wearable E-Skin and E-Textile Devices. Adv. Mater. 2019, 31, 1901408.

(6) Peng, H. Fiber-Shaped Energy Harvesting and Storage Devices. 1 ed.; Springer-Verlag Berlin Heidelberg: Heidelberg, 2015; pp. X, 218.

(7) Ryan, J. D.; Mengistie, D. A.; Gabrielsson, R.; Lund, A.; Müller, C. Machine-Washable PEDOT:PSS Dyed Silk Yarns for Electronic Textiles. ACS Appl. Mater. Interfaces 2017, 9, 9045-9050.

(8) Lam Po Tang, S. Recent Developments in Flexible Wearable Electronics for Monitoring Applications. Trans. Inst. Meas. Control 2016, 29, 283-300. 
(9) Zeng, W.; Shu, L.; Li, Q.; Chen, S.; Wang, F.; Tao, X.-M. FiberBased Wearable Electronics: A Review of Materials, Fabrication, Devices, and Applications. Adv. Mater. 2014, 26, 5310-5336.

(10) Ghahremani Honarvar, M.; Latifi, M. Overview of Wearable Electronics and Smart Textiles. J. Text. Inst. 2016, 108, 631-652.

(11) Rajan, K.; Garofalo, E.; Chiolerio, A. Wearable Intrinsically Soft, Stretchable, Flexible Devices for Memories and Computing. Sensors 2018, 18, 367.

(12) Abdelkader, A. M.; Karim, N.; Vallés, C.; Afroj, S.; Novoselov, K. S.; Yeates, S. G. Ultraflexible and Robust Graphene Supercapacitors Printed on Textiles for Wearable Electronics Applications. $2 D$ Materials 2017, 4, No. 035016.

(13) Guo, Y.; Otley, M. T.; Li, M.; Zhang, X.; Sinha, S. K.; Treich, G. M.; Sotzing, G. A. PEDOT:PSS "Wires" Printed on Textile for Wearable Electronics. ACS Appl. Mater. Interfaces 2016, 8, 2699827005.

(14) Yeo, J.; Kim, G.; Hong, S.; Kim, M. S.; Kim, D.; Lee, J.; Lee, H. B.; Kwon, J.; Suh, Y. D.; Kang, H. W.; Sung, H. J.; Choi, J.-H.; Hong, W.-H.; Ko, J. M.; Lee, S.-H.; Choa, S.-H.; Ko, S. H. Flexible Supercapacitor Fabrication by Room Temperature Rapid Laser Processing of Roll-to-Roll Printed Metal Nanoparticle Ink for Wearable Electronics Application. J. Power Sources 2014, 246, 562568.

(15) Liang, J.; Tong, K.; Pei, Q. A Water-Based Silver-Nanowire Screen-Print Ink for the Fabrication of Stretchable Conductors and Wearable Thin-Film Transistors. Adv. Mater. 2016, 28, 5986-5996.

(16) Lund, A.; Darabi, S.; Hultmark, S.; Ryan, J. D.; Andersson, B.; Ström, A.; Müller, C. Roll-to-Roll Dyed Conducting Silk Yarns: A Versatile Material for E-Textile Devices. Adv. Mater. Technol. 2018, 3, 1800251.

(17) Stoppa, M.; Chiolerio, A. Wearable Electronics and Smart Textiles: A Critical Review. Sensors 2014, 14, 11957-11992.

(18) Lamberti, A.; Gigot, A.; Bianco, S.; Fontana, M.; Castellino, M.; Tresso, E.; Pirri, C. F. Self-Assembly of Graphene Aerogel on Copper Wire for Wearable Fiber-Shaped Supercapacitors. Carbon 2016, 105, 649-654.

(19) Štefečka, M.; Kando, M.; Matsuo, H.; Nakashima, Y.; Koyanagi, M.; Kamiya, T.; Cernák, M. Electromagnetic Shielding Efficiency of Plasma Treated and Electroless Metal Plated Polypropylene Nonwoven Fabrics. J. Mater. Sci. 2004, 39, 2215-2217.

(20) Zabetakis, D.; Dinderman, M.; Schoen, P. Metal-Coated Cellulose Fibers for Use in Composites Applicable to Microwave Technology. Adv. Mater. 2005, 17, 734-738.

(21) Tebyetekerwa, M.; Xu, Z.; Li, W.; Wang, X.; Marriam, I.; Peng, S.; Ramkrishna, S.; Yang, S.; Zhu, M. Surface Self-Assembly of Functional Electroactive Nanofibers on Textile Yarns as a Facile Approach toward Super Flexible Energy Storage. ACS Appl. Energ. Mater. 2018, 1, 377-386.

(22) Yin, Z.; Jian, M.; Wang, C.; Xia, K.; Liu, Z.; Wang, Q.; Zhang, M.; Wang, H.; Liang, X.; Liang, X.; Long, Y.; Yu, X.; Zhang, Y. SplashResistant and Light-Weight Silk-Sheathed Wires for Textile Electronics. Nano Lett. 2018, 18, 7085-7091.

(23) Shao, W.; Tebyetekerwa, M.; Marriam, I.; Li, W.; Wu, Y.; Peng, S.; Ramakrishna, S.; Yang, S.; Zhu, M. Polyester@Mxene NanofibersBased Yarn Electrodes. J. Power Sources 2018, 396, 683-690.

(24) Liu, X.; Chang, H.; Li, Y.; Huck, W. T. S.; Zheng, Z. Polyelectrolyte-Bridged Metal/Cotton Hierarchical Structures for Highly Durable Conductive Yarns. ACS Appl. Mater. Interfaces 2010, 2, 529-535.

(25) Jur, J. S.; Sweet, W. J., III; Oldham, C. J.; Parsons, G. N. Atomic Layer Deposition of Conductive Coatings on Cotton, Paper, and Synthetic Fibers: Conductivity Analysis and Functional Chemical Sensing Using "All-Fiber" Capacitors. Adv. Funct. Mater. 2011, 21, 1993-2002.

(26) Shi, Z.; Phillips, G. O.; Yang, G. Nanocellulose Electroconductive Composites. Nanoscale 2013, 5, 3194-3201.

(27) Little, B. K.; Li, Y.; Cammarata, V.; Broughton, R.; Mills, G. Metallization of Kevlar Fibers with Gold. ACS Appl. Mater. Interfaces 2011, 3, 1965-1973.
(28) Lund, A.; van der Velden, N. M.; Persson, N.-K.; Hamedi, M. M.; Müller, C. Electrically Conducting Fibres for E-Textiles: An Open Playground for Conjugated Polymers and Carbon Nanomaterials. Mater. Sci. Eng. R Rep. 2018, 126, 1-29.

(29) Mirabedini, A.; Foroughi, J.; Wallace, G. G. Developments in Conducting Polymer Fibres: From Established Spinning Methods toward Advanced Applications. RSC Adv. 2016, 6, 44687-44716.

(30) Allison, L.; Hoxie, S.; Andrew, T. L. Towards SeamlesslyIntegrated Textile Electronics: Methods to Coat Fabrics and Fibers with Conducting Polymers for Electronic Applications. Chem. Commun. 2017, 53, 7182-7193.

(31) Zhang, X.; Lu, W.; Zhou, G.; Li, Q. Understanding the Mechanical and Conductive Properties of Carbon Nanotube Fibers for Smart Electronics. Adv. Mater. 2019, 32, 1902028.

(32) Fang, B.; Chang, D.; Xu, Z.; Gao, C. A Review on Graphene Fibers: Expectations, Advances, and Prospects. Adv. Mater. 2019, 32, 1902664.

(33) Kim, H.; Lee, G.; Becker, S.; Kim, J.-S.; Kim, H.; Hwang, B. Novel Patterning of Flexible and Transparent Ag Nanowire Electrodes Using Oxygen Plasma Treatment. J. Mater. Chem. C 2018, 6, 9394-9398.

(34) Lee, J.-Y.; Connor, S. T.; Cui, Y.; Peumans, P. SolutionProcessed Metal Nanowire Mesh Transparent Electrodes. Nano Lett. 2008, 8, 689-692.

(35) Madaria, A. R.; Kumar, A.; Zhou, C. Large Scale, Highly Conductive and Patterned Transparent Films of Silver Nanowires on Arbitrary Substrates and Their Application in Touch Screens. Nanotechnology 2011, 22, 245201.

(36) Kim, S.; Kim, J.; Kim, D.; Kim, B.; Chae, H.; Yi, H.; Hwang, B. High-Performance Transparent Quantum Dot Light-Emitting Diode with Patchable Transparent Electrodes. ACS Appl. Mater. Interfaces 2019, 11, 26333-26338.

(37) Park, M.; Kim, W.; Hwang, B.; Han, S. M. Effect of Varying the Density of Ag Nanowire Networks on Their Reliability During Bending Fatigue. Scr. Mater. 2019, 161, 70-73.

(38) Hwang, B.; Yun, T. G. Stretchable and Patchable Composite Electrode with Trimethylolpropane Formal Acrylate-Based Polymer. Composites, Part B 2019, 163, 185-192.

(39) Seo, Y.; Hwang, B. Mulberry-Paper-Based Composites for Flexible Electronics and Energy Storage Devices. Cellulose 2019, 26, $8867-8875$

(40) Kim, S.; Lee, H.; Kim, D.; Ha, H.; Qaiser, N.; Yi, H.; Hwang, B. Ethylcellulose/Ag Nanowire Composites as Multifunctional Patchable Transparent Electrodes. Surf. Coat. Technol. 2020, 125898.

(41) Takamatsu, S.; Kobayashi, T.; Shibayama, N.; Miyake, K.; Itoh, T. Fabric Pressure Sensor Array Fabricated with Die-Coating and Weaving Techniques. Sens. Actuators, A 2012, 184, 57-63.

(42) Hamedi, M.; Forchheimer, R.; Inganäs, O. Towards Woven Logic from Organic Electronic Fibres. Nat. Mater. 2007, 6, 357-362.

(43) Lee, S.; Shin, S.; Lee, S.; Seo, J.; Lee, J.; Son, S.; Cho, H. J.; Algadi, H.; Al-Sayari, S.; Kim, D. E.; Lee, T. Ag Nanowire Reinforced Highly Stretchable Conductive Fibers for Wearable Electronics. Adv. Funct. Mater. 2015, 25, 3114-3121.

(44) Ma, R.; Kang, B.; Cho, S.; Choi, M.; Baik, S. Extraordinarily High Conductivity of Stretchable Fibers of Polyurethane and Silver Nanoflowers. ACS Nano 2015, 9, 10876-10886.

(45) Ashayer-Soltani, R.; Hunt, C.; Thomas, O. Fabrication of Highly Conductive Stretchable Textile with Silver Nanoparticles. Text. Res. J. 2015, 86, 1041-1049.

(46) Castellino, M.; Chiolerio, A.; Shahzad, M. I.; Jagdale, P. V.; Tagliaferro, A. Electrical Conductivity Phenomena in an Epoxy Resin-Carbon-Based Materials Composite. Compos. A Appl. Sci. Manuf. 2014, 61, 108-114.

(47) Chiolerio, A.; Sangermano, M. In Situ Synthesis of Ag-Acrylic Nanocomposites: Tomography-Based Percolation Model, Irreversible Photoinduced Electromigration and Reversible Electromigration. Mater. Sci. Eng., B 2012, 177, 373-380.

(48) Kim, S.; Yun, T. G.; Kang, C.; Son, M.-J.; Kang, J.-G.; Kim, I.H.; Lee, H.-J.; An, C.-H.; Hwang, B. Facile Fabrication of Paper-Based 
Silver Nanostructure Electrodes for Flexible Printed Energy Storage System. Mater. Des. 2018, 151, 1-7.

(49) Lee, C.; Kim, H.; Hwang, B. Fracture Behavior of Metal Oxide/ Silver Nanowire Composite Electrodes under Cyclic Bending. J. Alloys Compd. 2019, 773, 361-366.

(50) Hwang, B.; Kim, W.; Kim, J.; Lee, S.; Lim, S.; Kim, S.; Oh, S. H.; Ryu, S.; Han, S. M. Role of Graphene in Reducing Fatigue Damage in $\mathrm{Cu} / \mathrm{Gr}$ Nanolayered Composite. Nano Lett. 2017, 17, 4740-4745.

(51) Sun, Y.; Mayers, B.; Herricks, T.; Xia, Y. Polyol Synthesis of Uniform Silver Nanowires: A Plausible Growth Mechanism and the Supporting Evidence. Nano Lett. 2003, 3, 955-960.

(52) Hwang, B.; Shin, H.-A.-S.; Kim, T.; Joo, Y.-C.; Han, S. M. Highly Reliable Ag Nanowire Flexible Transparent Electrode with Mechanically Welded Junctions. Small 2014, 10, 3397-3404.

(53) Massonnet, N.; Carella, A.; Jaudouin, O.; Rannou, P.; Laval, G.; Celle, C.; Simonato, J.-P. Improvement of the Seebeck Coefficient of PEDOT:PSS by Chemical Reduction Combined with a Novel Method for Its Transfer Using Free-Standing Thin Films. J. Mater. Chem. C 2014, 2, 1278-1283.

(54) Ouyang, J.; Chu, C.-W.; Chen, F.-C.; Xu, Q.; Yang, Y. HighConductivity Poly(3,4-Ethylenedioxythiophene):Poly(Styrene Sulfonate) Film and Its Application in Polymer Optoelectronic Devices. Adv. Funct. Mater. 2005, 15, 203-208.

(55) Ryan, J. D.; Lund, A.; Hofmann, A. I.; Kroon, R.; SarabiaRiquelme, R.; Weisenberger, M. C.; Müller, C. All-Organic Textile Thermoelectrics with Carbon-Nanotube-Coated N-Type Yarns. ACS Appl. Energ. Mater. 2018, 1, 2934-2941.

(56) Allison, L. K.; Andrew, T. L. A Wearable All-Fabric Thermoelectric Generator. Adv. Mater. Technol. 2019, 4, 1800615.

(57) Elmoughni, H. M.; Menon, A. K.; Wolfe, R. M. W.; Yee, S. K. A Textile-Integrated Polymer Thermoelectric Generator for Body Heat Harvesting. Adv. Mater. Technol. 2019, 4, 1800708. 